\title{
Single and Multiple Random Walks on Random Lattices: Excitation Trapping and Annihilation Simulations
}

\author{
R. Kopelman, ${ }^{1}$ J. Hoshen, ${ }^{1}$ J. S. Newhouse, ${ }^{1}$ and P. Argyrakis ${ }^{2}$
}

\begin{abstract}
Random walk simulations of exciton trapping and annihilation on binary and ternary lattices are presented. Single walker visitation efficiencies for ordered and random binary lattices are compared. Interacting multiple random walkers on binary and ternary random lattices are presented in terms of trapping and annihilation efficiencies that are related to experimental observables. A master equation approach, based on Monte Carlo cluster distributions, results in a nonclassical power relationship between the exciton annihilation rate and the exciton density.
\end{abstract}

KEY WORDS: Exciton; annihilation; fusion; trapping; diffusion; random walk; energy transfer; master equation.

\section{INTRODUCTION}

In this work we present an overview of random walk simulations on mixed lattices, concentrating on our newest results of one random walker and interacting multiple random walkers. The motivation has been to interpret experimental findings on molecular crystals at low temperatures, in particular energy transfer mechanisms for the molecular electronic excited singlet and triplet states. ${ }^{(1,2)}$ Such states can be adequately described as excitons, and the motion of the excitons can be thought of as mimicking random walk motion. Related properties include the number of sites visited in an $n$-step walk, which can be related to transport properties on solid lattices.

Presented at the Symposium on Random Walks, Gaithersburg, MD, June 1982.

Supported by NIH Grant No. 2 R01 NS08116-14.

'Department of Chemistry, University of Michigan, Ann Arbor, Michigan 48109.

${ }^{2}$ Department of Physics, University of Crete, Iraklion, Crete, Greece, and Department of Chemistry, University of Michigan, Ann Arbor, Michigan 48109. 
Since our crystals are purposely doped we deal primarily with binary and ternary systems in which random walk is allowed only on one component (open sites or donor sites) while a second component is inaccessible to the random walk (closed sites) resulting in scattering (reflection) each time the random walker reaches such sites. The third component serves the purpose of trapping and immobilizing the random walker if it happens to fall into such sites (acceptor sites). The three types of lattice sites are distinguished by their energy levels, the scatterer sites having the highest energy, the acceptor sites the lowest, and the donor sites being in the middle. A good example is the naphthalene system, ${ }^{(1,2)} \mathrm{C}_{10} \mathrm{D}_{8} / \mathrm{C}_{10} \mathrm{H}_{8} / \mathrm{BMN}$ (scatterer/ donor/acceptor) which has an effective square lattice topology (four nearest neighbors).

Even though the theory of random walks has been advanced recently, ${ }^{(3)}$ such complex systems as ours are still difficult to handle if one is looking for a closed form solution for the several random walk properties mentioned above. We, therefore, resorted to computer simulations that can accommodate all the complexities described and provide a reasonable quantitative picture of the problem we are investigating.

\section{FORMALISM AND RELATION TO EXPERIMENTS}

For a system in which energy is transferred on donor sites and trapped in acceptor sites a rigorous kinetic scheme describing the time evolution of these states can be written $a^{(4-6)}$

$$
\begin{aligned}
& \frac{d D}{d t}=-k_{d} D-k(t) D \\
& \frac{d A}{d t}=-k_{a} A-k(t) D
\end{aligned}
$$

where the only assumption is that there is no correlation between the natural decay and all other processes. Here $D$ and $A$ are the donor and acceptor populations, $k_{d}$ and $k_{a}$ are their natural decay rates (including both radiative and nonradiative processes but no energy transfer), and $k(t)$ is the time-dependent energy transfer rate coefficient. It has been shown that the probability for trapping by acceptor sites, $P(t)$, is given by ${ }^{(15)}$

$$
P(t)=P_{\infty}\left(1-e^{-S_{n} C_{a}}\right)+C_{a} I_{\mathrm{av}}
$$

where $C_{a}$ is the acceptor concentration relative to the donor concentration, $I_{\mathrm{av}}$ is the average finite donor cluster size in the binary scatterer-donor lattice, $P_{\infty}$ is the percolation probability of the donor lattice, and $S_{n}$ is the number of donor sites visited in an $n$-step walk. Well above the critical percolation concentration the second term goes to zero, and defining 
$\epsilon=S_{n} / n$ and $a=C_{a} n / t$ (where $t=$ time) we obtain

$$
P(t)=P_{\infty}\left(1-e^{-a t t}\right)
$$

The parameter $\epsilon$ we call the efficiency of the random walk. We can also rigorously show ${ }^{(5,6)}$ that

$$
k(t)=\frac{1}{1-P(t)} \frac{d P(t)}{d t}
$$

After inserting $P(t)$ from above, and setting $P_{\infty}=1$, which is valid well above the critical percolation concentration, we obtain

$$
k(t)=a\left(\epsilon+t \frac{d \epsilon}{d t}\right)
$$

One can now substitute the value of $k(t)$ in the original differential equation to obtain numerical solutions. Notice that all information regarding the proposed model of transfer has to come in this scheme only through $\epsilon$. We use random walk simulations to estimate $\epsilon$. The donor and acceptor time evolution curves can then be compared to experimental results of the time dependence of these states. ${ }^{(6)}$

In an alternative steady-state approach ${ }^{(7)}$ we compute $P$ in Eq. (3) for the overall lifetime, $\tau, P(\tau)$. The corresponding experimental quantity is the fraction $P=A /(A+D)$ which can be measured from relative donor and acceptor fluorescence intensities. Again, we see that the $P(\tau)$ quantity depends on $S_{n}$, a quantity that can be derived via random walk methods. A comparison with the experimental $P$ can then be made. (7)

Finally, a different experimental situation arises when a high density of excited states is generated, making it necessary to take into account interactions such as the fusion or annihilation of two excitons. The rates of such events as well as the overall behavior can be measured experimentally. ${ }^{(11)}$ We thus studied interacting multiple random walkers.

\section{RANDOM WALK SIMULATIONS}

\subsection{One Random Walker}

In the past we reported results ${ }^{(8)}$ for random walks on binary randomly mixed lattices, usually expressed in terms of the efficiency $\epsilon(\epsilon$ $=S_{n} / n, S_{n}$ being the number of sites visited in an $n$-step walk). Our model included a parameter $\ell$, the mean free path of the walk, over which there is a retention of directional memory after each step. For $l=1$ this is the case of an uncorrelated walk while when $l>1$ we have a correlated walk. The 
quantity $l$ is a measure of the "coherence" of the excited state in our physical system.

Summarizing our old results, we showed ${ }^{(8)}$ that as the donor/scatterer ratio decreases, $\epsilon$ also decreases, resulting in higher revisitation due to the increasing number of closed sites. Thus $\epsilon$ drops from $\epsilon=0.225\left(C_{d}=1.0\right)$ to $\epsilon=0.049\left(C_{d}=0.65\right)$; see Fig. 1. Also, we showed that for high donor concentrations, high $l$ values $(l \geqslant 10)$ give a higher efficiency than $l=1$. For $l=10$ and $C_{d}=1.0, \epsilon=0.630$, as opposed to $\epsilon=0.225$ for $l=1$. But closer to the critical percolation concentration, $l=1$ results in better efficiency than higher $l$ values. For $l=10$ and $C_{d}=0.65, \epsilon=0.010$ as opposed to $\epsilon=0.049$ for $l=1$. There is a crossover concentration where the two cases are about equal in efficiency, and for $l=1$ and $l=10$ it was shown ${ }^{(8)}$ that this occurred around $C_{d}=0.75$.

We now want to report on simulations performed on binary but ordered lattices. In these cases the closed sites (scatterers), instead of being randomly distributed, now occupy a rather ordered structure, the lattice now possessing some type of symmetry. Our results in Fig. 1 show that the efficiency is the same as for the random lattice above $C_{d}=0.90$. However, at intermediate concentrations, random walks on ordered lattices are considerably more efficient than on random ones. In these simulations the ordered lattices were generated giving the highest possible symmetry in each configuration. The same trend is observed for both high and low values of the parameter $l$. A more thorough discussion of this point can be found elsewhere. ${ }^{(9)}$

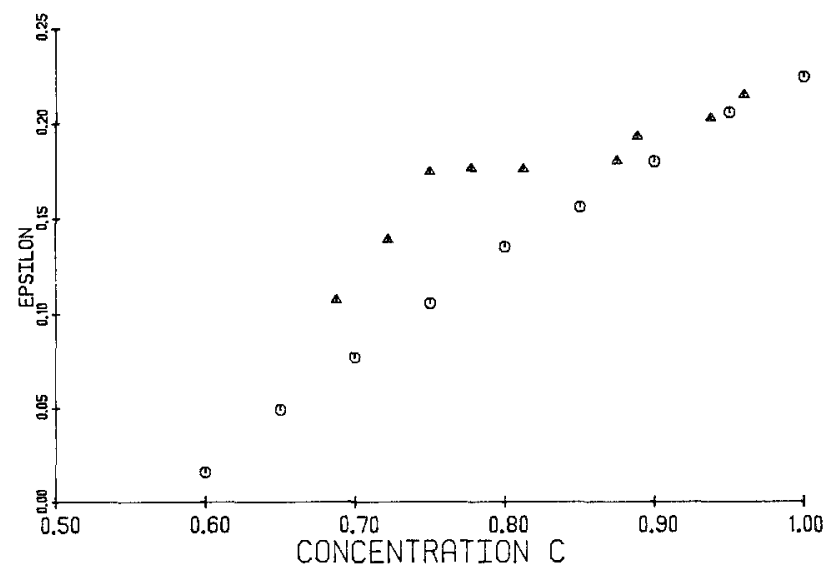

Fig. 1. Limiting efficiency $\epsilon$ at $n=200,000$ steps as a function of the donor sites concentration $C$, for random (circles) and ordered (triangles) lattices. 


\subsection{Multiple Interacting Random Walkers}

In this model ${ }^{(10)}$ several random walkers exist on a particular lattice at the same time. They are either generated via a continuous creation source or via a pulsed source. One step corresponds to one time unit. The interactions are included as follows: When two (or more) random walkers happen to occupy the same site, they are annihilated and deleted from the system. If a random walker falls into a trap site then it becomes immobilized and cannot be detrapped. If by the end of its lifetime no annihilation has occurred to a random walker, then it decays naturally. Random walkers that are trapped can also be annihilated if they are found at any instant occupying the same trap site. After some time $t$ a steady state is achieved as is shown in Fig. 2. In Fig. 3 we plot the rate of annihilation for a pulsed excitation and, as expected, we see a typical decay behavior. In Fig. 4 we plot several fractions ("efficiencies") for the annihilation $A$ and decay $D$ events versus the donor concentration. They all show the same picture, i.e., below the critical percolation concentration $P_{c}$, most random walkers are localized in isolated and relatively small clusters and cannot easily be trapped or annihilated. However, above $P_{c}$ the opposite is true and trapping and annihilation predominate.

In Fig. 5 we plot the decay rate, annihilation rate, and their ratio, for a wide concentration range and we see how the annihilation/decay rate fraction dramatically increases above the critical concentration $C_{d}=0.59$. Details of these calculations will be given elsewhere.

We are interested whether the annihilation rate as a function of the



Fig. 2. "Steady-state" population of excitons (random walkers) as a function of time (number of steps). Simulations were performed on a $200 \times 200$ square lattice with donor concentration $C_{d}=1.0$, acceptor concentration $C_{a}=1 \times 10^{-3}$ (mole fraction in donor lattice), an exciton interarrival time of 25 steps, and a lifetime of 2500 steps. 


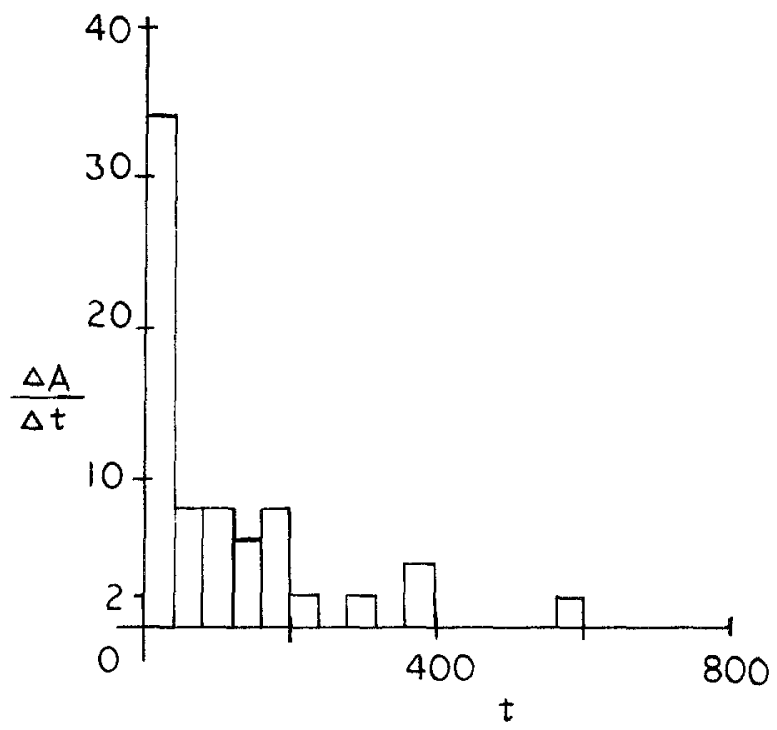

Fig. 3. Rate of annihilation as a function of time. Results of simulation on a $100 \times 100$ square lattice with donor concentration $C_{d}=1.00$, acceptor concentration $C_{a}=0.0$. All excitation is generated by a pulsed source at $t=0$, and the lifetime is 2500 steps.

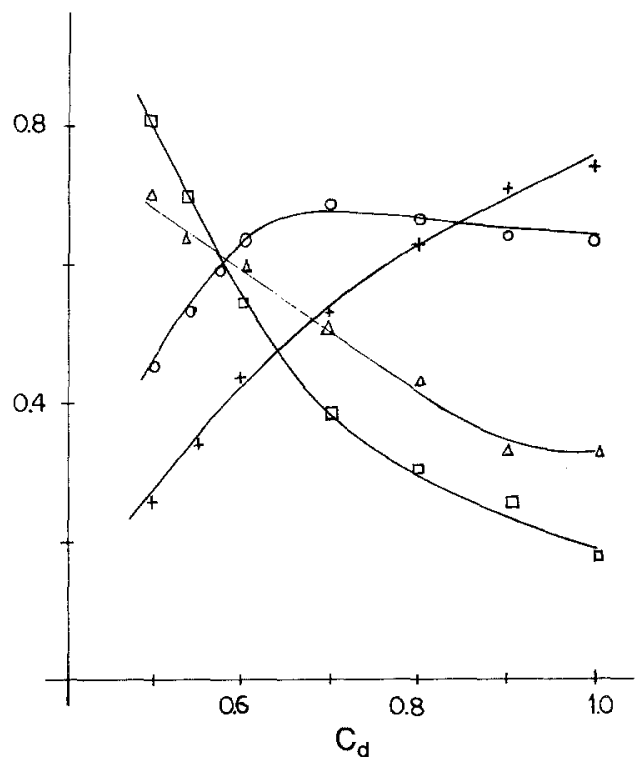

Fig. 4. Plot of several annihilation and decay fractions as a function of the donor concentration $C_{d}$. These are all performed on a $100 \times 100$ square lattice, with acceptor concentration $C_{a}=5 \times 10^{-3}$, lifetime of 5000 steps, and interarrival time of 100 steps. Circles: $\left(A_{d}+A_{a}\right)$ $/\left(A_{a}+A_{d}+D_{d}+D_{a}\right)$, squares: $D_{d} /\left(D_{d}+D_{a}\right)$, triangles: $A_{d} /\left(A_{d}+A_{a}\right)$, crosses: $\left(A_{a}+D_{a}\right)$ $/\left(A_{\alpha}+A_{d}+D_{\alpha}+D_{d}\right)$. The solid lines are for visual purposes only. Note that here $A$ and $D$ designate annihilation and decay events, respectively, while $a$ and $d$ designate acceptor and donor sites, respectively. 


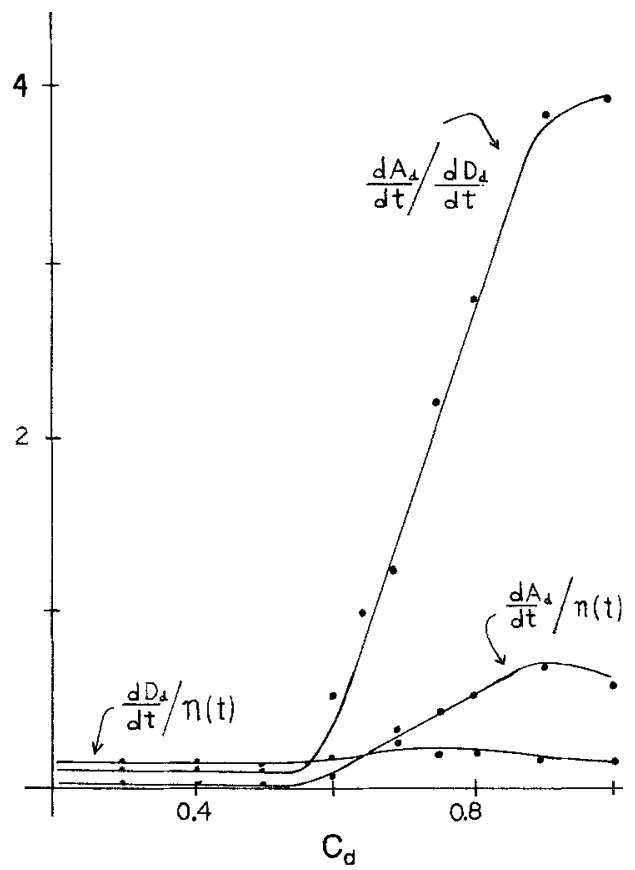

Fig. 5. Plot of annihilation and decay rates as a function of donor concentration $C_{d}$. Results of simulations on a $100 \times 100$ square lattice with $C_{a}=0.0$, exciton interarrival time of 100 steps, and lifetime of 5000 steps. The rates were computed after 19000 steps. We show the decay rate $d D_{d} / d t$ (normalized by the number of excitons present), the annihilation rate, $d A_{d} / d t$, and the annihilation/decay rate ratio, $\left(d A_{d} / d t\right) /\left(d D_{d} / d t\right)$. Notation is the same as for Fig. 4.

excitation population gives a power law of 2 , over the entire donor concentration range. Having found experimental behavior ${ }^{(1)}$ that consistently deviates from such a power law of 2 we turn to a different approach and use a master equation that includes annihilation and decay events. ${ }^{(12)}$ The master equation describing this system is (see definitions in ref. 12)

$$
\begin{aligned}
d p_{n}(t) / d t= & \beta\left(L / L_{0}\right) p_{n-1}(t)-\beta\left(L / L_{0}\right) p_{n}(t)+(n+1) \beta p_{n+1}(t) \\
& -n \beta p_{n}(t)+\left[\left(G_{n+1} L_{c} \beta\right) / L^{2}\right] p_{n+1}(t)-\left[\left(G_{n} L_{c} \beta\right) / L^{2}\right] p_{n}(t)
\end{aligned}
$$

where the first two terms give the excitation rate, the next two terms are the first-order rates of depopulating the $(n+1)$ and the $n$ levels, and the last two terms give the analogous second-order rates. From cluster distribution 
calculations ${ }^{(14)}$ we are now in a position to account for the occupation of all clusters in a binary lattice and estimate all the pertinent occupation probabilities. Using a cluster multiple labeling technique we have recently derived cluster distributions for lattices of over one billion $\left(10^{9}\right)$ sites. However, these results will be reported elsewhere. ${ }^{(16)}$ An adequate solution to the master equation can be attained using lattices of the order of $10^{5}$ sites.

The solution to Eq. (7) for the probabilities $p_{n}^{0}$ are given by

$$
p_{n}^{0}=\left(L / L_{0} K\right) p_{n-1}^{0}
$$

where

$$
K_{n}=n+G_{n} L_{c} / L^{2}
$$

It can finally be shown that the expectation value of the exciton population $n(t)$ and annihilation rate $I(t)$ are given by

$$
\begin{aligned}
& \langle n(t)\rangle=\sum_{n=1} n p_{n}(t) \\
& \langle I(t)\rangle=\sum_{n=1}\left(G_{n} L_{c} / L^{2}\right) p_{n}(t)
\end{aligned}
$$

Both $\langle n(t)\rangle$ and $\langle I(t)\rangle$ can now be calculated for specific lattices, rate constants, and excitation densities. Finally, from the $\langle I(t)\rangle$ versus $\langle n(t)\rangle$ plot (in logarithmic scale) we can derive the expected power law from the line slope. This was performed for a wide range of concentrations and the results $^{(17)}$ are shown in Fig. 6 . We see that there is no deviation from the

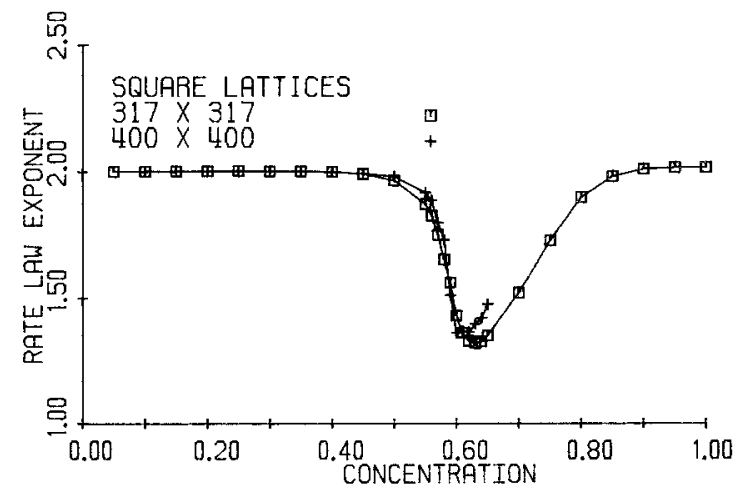

Fig. 6. Power law behavior as a function of the donor concentration. Based on the annihilation rate and excitation population given by the solutions of the master equation (see text for discussion). $L_{c}=\gamma L$, where $\gamma=10^{5}$. 
power of 2 well below and well above the critical percolation concentration, but around that region the plot shows a minimum of about 1.3. We intend to investigate this problem further via annihilation simulations.

\section{REFERENCES}

1. P. Argyrakis, Doctoral dissertation, University of Michigan (1979).

2. R. Kopelman, in Modern Problems in Solid State Physics, Vol 00: Spectroscopy and Excitation Dynamics of Condensed Molecular Systems, V. M. Agranovich and R. M. Hochstrasser, eds. (North-Holland, Amsterdam, 1982).

3. See Proceedings of the Symposium on random walks and their application to the physical and biological sciences, Washington, DC, J. Stat. Phys, 00(1982).

4. R. C. Powel and Z. G. Soos, J. Lumin. 11:1 (1975).

5. R. Kopelman and P. Argyrakis, J. Chem. Phys. 72:3053 (1980).

6. P. Argyrakis and R. Kopelman, Chem. Phys. Lett. 61:187 (1979); and to be published.

7. P. Argyrakis and R. Kopelman, Chem. Phys. 57:29 (1981).

8. P. Argyrakis and R. Kopelman, Phys. Rev. B 22: 1830 (1980).

9. P. Argyrakis, Phys. Rev. B 26 (to appear) (1982).

10. P. Argyrakis, J. Hoshen, and R. Kopelman in Fast Reactions in Energetic Systems, C. Capellos and R. Walker, eds. (Reidel, Dordrecht, 1981) p. 685.

11. P. W. Klymko and R. Kopelman, J. Lumin. 24/25:457 (1981); P. W. Klymko and R. Kopelman, J. Phys. Chem., 86:3686 (1982).

12. S. E. Webber and C. E. Swenberg, Chem. Phys. 49:231 (1980).

13. J. Hoshen, P. Klymko, and R. Kopelman, J. Stat. Phys. 21:583 (1979).

14. J. Hoshen, R. Kopelman, and E. M. Monberg, J. Stat. Phys. 19:219 (1978).

15. J. Hoshen and R. Kopelman, J. Chem. Phys. 65:2817 (1976).

16. J. Hoshen, J. S. Newhouse, and R. Kopelman (unpublished).

17. P. W. Klymko, J. S. Newhouse, and R. Kopelman (unpublished). 